\title{
Dually responsive one dimensional photonic crystals with reversible color changes
}

\author{
Cihui Liu ${ }^{a}$, Chong Yao ${ }^{a}, Y_{a n x i} Z_{h}^{a}{ }^{,}$Jiaoyu Ren $^{a}$, and Liqin Ge ${ }^{a}$, \\ ${ }^{a}$ State Key Laboratory of Bioelectronics, School of Biological Science and Medical \\ Engineering, Southeast University, Nanjing 210096, P.R. China
}


ABSTRACT: We developed a smart and reproducible fabrication of $\mathrm{pH} /$ temperature dually responsive sensor by taking advantage of one dimensional photonic crystals (1DPCs). $\mathrm{TiO}_{2} / \mathrm{P}\left(\mathrm{AA}-\right.$ bis-NiPAAm) 1 DPCs were fabricated by spin-coating $\mathrm{TiO}_{2}$ and copolymers P(AA-bis-NiPAAm) onto silicon wafers. When using the 1DPCs dually responsive polymer films as $\mathrm{pH} /$ temperature sensor, both refraction wavelength and peak refraction intensity values were derived, and the reproducible change could be observed by the naked eye through a change in color. 
KEYWORDS: 1DPCs, dually responsive, $\mathrm{P}\left(\mathrm{NiPAAm}-\right.$ bis-AA), $\mathrm{TiO}_{2}$ 


\section{Introduction}

Photonic crystal hydrogel sensor exhibits "structural color" due to the selective diffraction of light from periodic structures of the hydrogel.[1-5] Binding of analyte to the functional group of the hydrogel causes osmotic pressure increase in the hydrogel, and the following volume change of the hydrogel sensor driven by water influx to compensate the osmotic pressure directly induces a change in structural color.[6-12] The reversible sensing behavior has potential applications in chemical and biological sensors.

Due to this reversible color tunability by external stimuli, the hydrogel sensor can be categorized as a smart photonic crystal.[13] Two of the most attractive features of the photonic crystal hydrogel sensors are easy recognition of stimuli by naked eye without necessity of complex detection systems, and reusability of the sensor as long as the stimuli binding to the hydrogel is a reversible process.[14] As a typical example, acrylic monomer containing the sensing moiety is copolymerized with hydrogel monomer such as 2-hydroxyethyl methacrylate (HEMA) and a suitable cross-linker.[15, 16] Various stimuli-responsive hydrogel sensors can be fabricated by changing the monomers.[17-19] Gu and coworkers demonstrated tunable photonic crystals beads composed of ethoxylated trimethylolpropane triacrylate (ETPTA) and polyethylene glycol (PEG) for multifunctional biomedical applications.[20] Ever since the pioneering work reported by some researchers, there have been lots of investigations on photonic crystal hydrogel sensors for such as $\mathrm{pH}[16,21]$, humidity,[22] temperature,[23] metal ions,[24, 25] glucose,[26] and so on.[27]

By introducing more than one sensing moieties into the hydrogel system, multi-response hydrogel sensors can be produced. Takeoka and coworkers reported on a dual tunable sensor for thermo and pH by copolymerizing methacrylic acid (MAA) and N-isopropylacrylamide (NiPAAm).[28] 
Zhang and coworkers demonstrated a glucose-temperature dual sensor.[29] Takeoka and coworkers also fabricated photochromic hydrogel sensor which responses simultaneously to the UV-light and temperature by copolymerizing NiPAAm and azobenzene-containing acrylic monomer where azobenzene unit in the hydrogel induced volume change through the photo isomerization process.[30] Among this research works, NiPAAm is a well-known functional hydrogel which exhibits a thermo responsive volume transition by lower critical solution temperature (LCST) behavior at about $32{ }^{\circ} \mathrm{C}$. Below LCST, NiPAAm swells by being compatible with water while it collapses over LCST due to the hydrophobic transition.

Sensing materials for temperature, $\mathrm{pH}$, redox-active compounds, ionic species, glucose, solvents, light, magnetic fields, and electric fields have been prepared in 3D photonic crystals dual-responsive systems.[31] When turn the attention to 1DPC, it provides easy fabrication process, ordered stack structure with controllable composite layer thickness and controllable responsiveness.[32, 33] Incorporation of functional nano-materials within 1DPCs template is regarded as a clever idea toward many useful applications such as colorimetric sensors,[34, 35] solar cell,[36] and high index photonic band gap materials.[37, 38] Among those, responsive 1DPCs with stimuli-response hydrogel have drawn a growing interest over the last decade due to its potential toward various "smart" sensor applications.[32, 39-44] However, there have been few reports on dual-responsive hydrogel 1DPCs which can independently and extensively response to two stimulus.

In this study, we present a smart and reproducible fabrication of $\mathrm{pH} /$ temperature dually responsive sensor with rapid responses by copolymerizing AA and NiPAAm within the $\mathrm{TiO}_{2} / \mathrm{P}(\mathrm{AA}-\mathrm{bis}-\mathrm{NiPAAm})$ 1DPCs structure which have ordered stack structure, controllable 
composite layer thickness and controllable responsiveness. (see Figure 1). In addition to the responsiveness investigation, the reversibility of the sensor responses upon consecutive $\mathrm{pH}$ and temperature variations are also demonstrated. These features make 1DPCs ideal for sensing applications.

\section{Materials and methods}

\subsection{Materials}

As monomers for hydrogel, acrylic acid (AA, Sigma Aldrich), N-isopropylacrylamide (NiPAAm, Sigma Aldrich) were used. N, N'-methylene-bis-acrylamide (BIS, Sigma Aldrich) and ammonium persulfate (APS; Sigma Aldrich) was used as crosslinker, and thermal initiator respectively. 9.72 mmol NIPAm, $0.48 \mathrm{mmol}$ of AA, $0.81 \mathrm{mmol}$ of BIS, $0.29 \mathrm{mmol}$ of APS, and $34.7 \mathrm{mmol}$ of DI water were mixed. Synthesis of the $\mathrm{TiO}_{2}$ Sol: Tetrabutyl titanate $(20 \mathrm{~g})$ was dissolved in $20 \mathrm{~g}$ of ethyl alcohol with stirring for $1 \mathrm{~h}$. Acetic acid (2.12 g), hydrochloric acid (4.06 g), and ethanol (7 g) were then mixed homogeneously followed by adding dropwise into the above solution and stirred for another $3 \mathrm{~h}$. The obtained yellow transparent solution was allowed to undergo hydrolysis reaction at room temperature overnight. The phosphate buffer solutions of various $\mathrm{pH}$ were prepared by mixing $0.1 \mathrm{M}$ aq. $\mathrm{KH}_{2} \mathrm{PO}_{4}$ with $\mathrm{HCl}$ or $\mathrm{NaOH}$.

\subsection{Fabrication of dually responsive hydrogel 1DPCs}

The 1DPCs were fabricated by spin-coating the $\mathrm{TiO}_{2}$ sol and PNiPAAm-bis-AA alternately; both the $\mathrm{TiO}_{2}$ sol and the polymer precursor were spin-coated at $3000 \mathrm{rpm}$ for $30 \mathrm{~s}$ onto a silicon wafer. In order to get different film thicknesses, compositions can be diluted into different concentrations or spin-coated in different rotate speeds. The $\mathrm{TiO}_{2}$ layer was baked at $60{ }^{\circ} \mathrm{C}$ for $10 \mathrm{~min}$ and the polymer layer was baked at $135{ }^{\circ} \mathrm{C}$ for $10 \mathrm{~min}$. The first layer was $\mathrm{TiO}_{2}$ layer and the last layer 
was polymer layer in all our experiments. The total number of the layers is 6 , which means there is 3 bilayers structure.

\subsection{Measurements of sensing responses}

Scanning electron microscopy (SEM) images were obtained from a field emission scanning electron microscope (FE-SEM, HitachiS-3000N, Japan). In situ reflectance spectra were collected by a fiber optic spectrophotometer (Ocean Optics, Inc., HR 2000256 UV-Vis-NIR). Angle dependence intensity spectra were obtained by Spectroscopic Ellipsometry (Semilab GES5E). Color photos were taken on a digital camera (Canon5D Mark II, Japan). Measurement of sensor response carried out by collecting a series of reflectance during $\mathrm{pH}$ or temperature changes. For temperature/pH dependent measurement, a sample was placed in a home-made vessel.

\section{Results and discussion}

The smart and reproducible fabrication of $\mathrm{pH} /$ temperature dually responsive 1DPCs were achieved through alternating $\mathrm{TiO}_{2}$ and $\mathrm{P}\left(\mathrm{NiPAAm}-\right.$ bis-AA) by spin-coating. $\mathrm{TiO}_{2}$ layer is chosen because of its high refractive index compared with polymer layer and stable structure in sensing process. Furthermore, it can form uniform film easily. The effective refractive indexes of the $\mathrm{P}\left(\mathrm{NiPAAm}-\right.$ bis-AA) and $\mathrm{TiO}_{2}$ layers were determined by spectroscopic ellipsometry to be 1.585 and 1.831 respectively.

\subsection{Optical properties of 1DPCs}

Tuning of optical properties of 1DPCs can be achieved by increasing the number of layers, which is shown in Figure 2. An intense and wide Bragg peak (with peak position at $575 \mathrm{~nm}$ ) is observed, which is a direct consequence of the large refractive index contrast between the $\mathrm{TiO}_{2}$ layer and polymer layer. The photonic band width becomes narrower and the intensity of the peak grows 
with increasing numbers of layers, which are in good agreement with previous theory of the thickness dependence of the optical response of photonic crystal slabs, Fig. 2(a).

A change of the layer thickness also influences the optical properties of 1DPCs. Increasing the layer thickness, the position of band gap will make a red shift due to Bragg-Snell's law, see in supporting information. [45] According to the significant refractive indices difference between two components, the obvious photonic stopband and structural color can be easily obtained in several bilayers. As shown in the reflective spectra (Figure 2b), fixing the thickness of $\mathrm{TiO}_{2}$ layer at a proper thickness, while changing the thickness of polymer layer increasingly, the Bragg peak positions are at $461 \mathrm{~nm}, 520 \mathrm{~nm}, 571 \mathrm{~nm}$ and $626 \mathrm{~nm}$ respectively, which agrees with the experiment results. We can see that the Bragg peak positions increase direct proportionably when increasing the thickness of one layer while keeping the other layer constant. The Bragg peak can be manipulated in the full visible range from blue to red by choosing proper periods. The film is very uniform over a large area, see in Fig. 2(c, d). In our experiments, color uniformity can be achieved over an area of $15 \mathrm{~mm} \times 15 \mathrm{~mm}$ at least. The 1DPCs can keep its color invariable for a long time, which indicates that the prepared materials and microstructures have perfect stability.

\subsection{Temperature and $\mathrm{pH}$ dually response sensing}

This dual-responsive 1DPC is composed of NiPAAm, acrylic acid (AA), and N, N'-methylene-bis-acrylamide (BIS). An extensively studied water soluble thermo responsive polymer is PNiPAAm, which undergoes a phase transition at about $32{ }^{\circ} \mathrm{C}$, expelling the majority of its solvating water in the process. The unique properties can be used to synthesize a variety of hydrogels with varying chemical and physical properties. The addition of co-monomers to the hydrogels can impact their responsivity and can have a strong impact on the photonic structure. 
One common co-monomer is acrylic acid (AA). As a monomer, AA has an acid dissociation constant (pKa) of about 4.25 at $25{ }^{\circ} \mathrm{C}$. Moreover, the pKa values of AA copolymerized in polymers are larger than 4.25 , as a result of electrostatic interaction between neighboring AA monomers.[21]

In Fig. 3, typical sensing responses upon temperature or $\mathrm{pH}$ stimuli are shown. The size of the $1 \mathrm{DPC}$ in the photographs is $15 \mathrm{~mm} \times 15 \mathrm{~mm}$. The reflectance spectrum of a $1 \mathrm{DPC}$ usually includes the reflection maxima at visible wavelength range which stands for the structural color of the sensor varying with external stimuli. Fig. 3(a) shows the reflectance spectra measured at six different temperatures under fixed $\mathrm{pH}$ of 4.0. At a fixed $\mathrm{pH}$, the temperature variation from $25{ }^{\circ} \mathrm{C}$ to $50{ }^{\circ} \mathrm{C}$ induced the band gap position changes from $600 \mathrm{~nm}$ to $505 \mathrm{~nm}$ as well as the colors of the 1DPCs change from red to green at different temperatures which are shown as photographs on bottom in Fig. 3(b).

Figure 4 shows the $\mathrm{pH}$-dependent reflectance spectra at seven different $\mathrm{pH}$ buffers under the fixed temperature of $35^{\circ} \mathrm{C}$. According to Figure 4, when a PNiPAAm-bis-AA is at about $\mathrm{pH} 7.0$ exhibits dramatic swelling power, resulting from both electrostatic repulsion between the charges on the polymer chains and osmotic pressure of the counterions.[46] Likewise, $\mathrm{pH}$ variation from 8.0 to 2.0 induced the band gap position changes from $575 \mathrm{~nm}$ to $510 \mathrm{~nm}$, Fig. 4(a), at a fixed temperature of $35^{\circ} \mathrm{C}$ induced the color change of the sensor from yellow to blue-green, Fig. 4(b)

The sensor responses upon $\mathrm{pH}$ and temperature stimuli can also be demonstrated by showing the changes of with respect to the stimuli conditions. Fig. 5a shows max reflection wavelength changes upon temperature variation measured at seven different $\mathrm{pH}$ buffers. It is worth noting that there are significant jumps of max reflection wavelength along with temperature curves between 
the $\mathrm{pH} 6.0$ and 7.0 since $\mathrm{pKa}$ of AA within the hydrogel where the $\mathrm{pH}$ sensitivity is maximized.[47] Since the 1DPCs sensors used in the current study well stacked on the silicon wafer substrate, we assumed that the swelling occurs only along the vertical direction to the substrate surface which changes the polymer layers' thickness. From the series of reflectance spectra obtained during both stimuli, time-dependent variations of reflection peak value were plotted during the initial stages of swelling as shown in Fig. 5b. After the $\mathrm{pH}$ driven transformation begins, the peak reflectance value gradually decreased as the refractive indices differences between water and the hydrogel became smaller. As the water infiltrate into the 1DPC structure, more swollen hydrogel generally possesses a lower refractive index than the shrunken state due to inclusion of water. The peak value of reflection get a decrease immediately. For T-variation case, however, the intensity of the peak sharply get a rise up within $20 \mathrm{~s}$ after which increasing reflectance was observed just opposite to the $\mathrm{pH}$ response. The initial rise and the following recovery of the reflection peak intensity can partly explain an exceptionally rapid response of the T-drop experiment. When T-variation is applied, the 1DPC film make a rapid color change which can be observed by naked eyes.

To confirm the performance of the PNiPAAm-bis-AA 1DPCs in practical applications, several circles of temperature and $\mathrm{pH}$ variation were tested. The reflectance spectra were recorded, and their peak wavelength values were collected for further analysis. The results were shown in Fig. 6. When fix the $\mathrm{pH}$ buffer at 4.0, Fig. 6a shows the reversible change in max of PNiPAAm-bis-AA 1DPC as temperature varies from $25^{\circ} \mathrm{C}$ to $50^{\circ} \mathrm{C}$. It can be seen that the PCs swell and deswell completely reversibly under different temperature. When fix the temperature at $35{ }^{\circ} \mathrm{C}$, Fig. $6 \mathrm{~b}$ shows the reversible change in max of PNiPAAm-bis-AA 1DPC as $\mathrm{pH}$ varies from 2.0 to 8.0. This 
fact further indicates that PNiPAAm-bis-AA chains are firmly embodied on the silicon wafer, and the 1DPC has an excellent durability. Considering that free radicals can be unpredictably reactive in condensed phases, some covalent cross-linking may happen. This may be the reason that the introduced PAA cannot be washed out. These two oscillation results show excellent reversibility for 10 cycles, respectively. With its responsiveness and durability, this novel modified 1DPC can be potentially used in fabricating chemical and biochemical sensors.

\section{Conclusion}

In summary, by combining with dually-responsive copolymer and 1DPC fabrication method, we have successfully introduced a $\mathrm{pH} /$ temperature dually responsive $\mathrm{P}\left(\mathrm{AA}-\right.$ bis-NiPAAm) $/ \mathrm{TiO}_{2} 1 \mathrm{DPC}$ affording a new type of stimuli-responsive optical material with quick response time. When using the 1DPCs dually responsive polymer films as $\mathrm{pH} /$ temperature sensor, both refraction wavelength and peak refraction intensity values were derived, and the reproducible change could be observed by the naked eye through a change in color. Furthermore, it could contribute to a multitude of 1DPCs, implying that it could find applications in various areas, such as chemical sensors, and environmental monitoring. 


\section{Acknowledgements}

The authors thank for the financial support of pre-research foundation for national science foundation and the Fundamental Research Funds for the Central Universities. This work is also supported by the fund for the analysis and testing of the large-scale apparatus from the Southeast

University. Prof. Dr. Ge also thanks for the support from Prof. Gu Zhongze. 


\section{Reference}

[1] P.G. O'Brien, D.P. Puzzo, A. Chutinan, L.D. Bonifacio, G.A. Ozin, N.P. Kherani, Selectively Transparent and

Conducting Photonic Crystals, Adv. Mater. 22 (2010) 611-616.

[2] Y.P. Zhang, V.P. Chodavarapu, A.G. Kirk, M.P. Andrews, Structured color humidity indicator from reversible pitch tuning in self-assembled nanocrystalline cellulose films, Sens. Actuators, B. Chem. 176 (2013) 692-697.

[3] Z. Wu, D. Lee, M. Rubner, R. Cohen, Structural Color in Porous, Superhydrophilic, and Self-Cleaning $\mathrm{SiO}_{2} / \mathrm{TiO}_{2}$ Bragg Stacks, Small, 3 (2007) 1467-1467.

[4] Z. Xie, H. Gu, C. Zhu, Z. Gu, Bio-inspired variable structural color materials, Chem. Soc. Rev. (2012).

[5] E. Tian, Y. Ma, L. Cui, J. Wang, Y. Song, L. Jiang, Color-Oscillating Photonic Crystal Hydrogel, Macromol. Rapid Commun. 30 (2009) 1719-1724.

[6] Y. Zhao, X. Zhao, B. Tang, W. Xu, J. Li, J. Hu, Z. Gu, Quantum-Dot-Tagged Bioresponsive Hydrogel Suspension Array for Multiplex Label-Free DNA Detection, Adv. Funct. Mater. 20 (2010) 976-982.

[7] Y.F. Yue, M.A. Haque, T. Kurokawa, T. Nakajima, J.P. Gong, Lamellar Hydrogels with High Toughness and Ternary Tunable Photonic Stop-Band, Adv. Mater. 25 (2013) 3106-3110.

[8] Z. Wang, J. Zhang, Z. Tian, Z. Wang, Y. Li, S. Liang, L. Cui, L. Zhang, H. Zhang, B. Yang, Organic-inorganic hybrid photonic hydrogels as a colorful platform for visual detection of SCN, Chem. Commun. 46 (2010) 8636-8638.

[9] E. Tian, J. Wang, Y. Zheng, Y. Song, L. Jiang, D. Zhu, Colorful humidity sensitive photonic crystal hydrogel, J. Mater. Chem. 18 (2008) 1116-1122.

[10] W. Shen, M. Li, C. Ye, L. Jiang, Y. Song, Direct-writing colloidal photonic crystal microfluidic chips by inkjet printing for label-free protein detection, Lab on a Chip, 12 (2012) 3089-3095.

[11] M. Li, F. He, Q. Liao, J. Liu, L. Xu, L. Jiang, Y. Song, S. Wang, D. Zhu, Ultrasensitive DNA Detection Using 
Photonic Crystals, Angew. Chem. Int. Ed. 47 (2008) 7258-7262.

[12] Q. Zhong, H. Xu, H. Ding, L. Bai, Z. Mu, Z. Xie, Y. Zhao, Z. Gu, Preparation of conducting polymer inverse opals and its application as ammonia sensor, Colloids Surf. A: Physicochem. Eng. Asp. 433 (2013) 59-63.

[13] Y. Zhao, X. Zhao, Z. Gu, Photonic Crystals in Bioassays, Advanced Functional Materials, 20 (2010) 2970-2988.

[14] J.E. Stumpel, D.J. Broer, A.P.H.J. Schenning, Stimuli-responsive photonic polymer coatings, Chem. Commun. $50(2014) 15839-15848$.

[15] Z. Wang, J. Zhang, J. Li, J. Xie, Y. Li, S. Liang, Z. Tian, C. Li, Z. Wang, T. Wang, H. Zhang, B. Yang, Colorful detection of organic solvents based on responsive organic/inorganic hybrid one-dimensional photonic crystals, J. Mater. Chem. 21 (2011) 1264-1270.

[16] X. Xu, A.V. Goponenko, S.A. Asher, Polymerized PolyHEMA Photonic Crystals: pH and Ethanol Sensor Materials, J. Am. Chem. Soc. 130 (2008) 3113-3119.

[17] E. Kim, C. Kang, H. Baek, K. Hwang, D. Kwak, E. Lee, Y. Kang, E.L. Thomas, Control of Optical Hysteresis in Block Copolymer Photonic Gels: A Step Towards Wet Photonic Memory Films, Adv. Funct. Mater. 20 (2010) $1728-1732$.

[18] P. Kang, S.O. Ogunbo, D. Erickson, High Resolution Reversible Color Images on Photonic Crystal Substrates, Langmuir, 27 (2011) 9676-9680.

[19] Y.J. Lu, H.W. Xia, G.Z. Zhang, C. Wu, Electrically tunable block copolymer photonic crystals with a full color display, J. Mater. Chem. 19 (2009) 5952-5955.

[20] Y. Zhao, Z. Xie, H. Gu, L. Jin, X. Zhao, B. Wang, Z. Gu, Multifunctional photonic crystal barcodes from microfluidics, NPG Asia Mater. 4 (2012) e25.

[21] M. Honda, T. Seki, Y. Takeoka, Dual Tuning of the Photonic Band-Gap Structure in Soft Photonic Crystals, 
Adv. Mater. 21 (2009) 1801-1804.

[22] J.-H. Kang, J.H. Moon, S.-K. Lee, S.-G. Park, S.G. Jang, S. Yang, S.-M. Yang, Thermoresponsive Hydrogel Photonic Crystals by Three-Dimensional Holographic Lithography, Adv. Mater. 20 (2008) 3061-3065.

[23] B. Brugger, J. Vermant, W. Richtering, Interfacial layers of stimuli-responsive poly-(N-isopropylacrylamide-co-methacrylicacid) (PNIPAM-co-MAA) microgels characterized by interfacial rheology and compression isotherms, Phys. Chem. Chem. Phys. 12 (2010) 14573-14578.

[24] Y. Huang, F. Li, M. Qin, L. Jiang, Y. Song, A Multi-stopband Photonic-Crystal Microchip for High-Performance Metal-Ion Recognition Based on Fluorescent Detection, Angew. Chem. Int. Ed. 52 (2013) 7296-7299.

[25] D. Arunbabu, A. Sannigrahi, T. Jana, Photonic crystal hydrogel material for the sensing of toxic mercury ions (Hg2+) in water, Soft Matter, 7 (2011) 2592.

[26] L. Jin, Y. Zhao, X. Liu, Y. Wang, B. Ye, Z. Xie, Z. Gu, Dual signal glucose reporter based on inverse opal conducting hydrogel films, Soft Matter, 8 (2012) 4911-4917.

[27] J. Hou, H. Zhang, Q. Yang, M. Li, Y. Song, L. Jiang, Bio-Inspired Photonic-Crystal Microchip for Fluorescent Ultratrace Detection, Angew. Chem. Int. Ed. 126 (2014) 5901-5905.

[28] K. Ueno, K. Matsubara, M. Watanabe, Y. Takeoka, An Electro- and Thermochromic Hydrogel as a Full-Color Indicator, Adv. Mater. 19 (2007) 2807-2812.

[29] S. Xing, Y. Guan, Y. Zhang, Kinetics of Glucose-Induced Swelling of P(NIPAM-AAPBA) Microgels, Macromolecules, 44 (2011) 4479-4486.

[30] K. Matsubara, M. Watanabe, Y. Takeoka, A Thermally Adjustable Multicolor Photochromic Hydrogel, Angew. Chem. Int. Ed. 46 (2007) 1688-1692.

[31] H. Cong, B. Yu, J. Tang, Z. Li, X. Liu, Current status and future developments in preparation and application 
of colloidal crystals, Chem. Soc. Rev. 42 (2013) 7774-7800.

[32] I. Pavlichenko, A.T. Exner, M. Guehl, P. Lugli, G. Scarpa, B.V. Lotsch, Humidity-Enhanced Thermally Tunable TiO2/SiO2 Bragg Stacks, J. Phys. Chem. C, 116 (2011) 298-305.

[33] G. von Freymann, V. Kitaev, B.V. Lotsch, G.A. Ozin, Bottom-up assembly of photonic crystals, Chem. Soc. Rev. (2013).

[34] C. Liu, C. Yao, Y. Zhu, J. Ren, K. Lan, H. Peng, L. Ge, Patterned one-dimensional photonic crystals with acidic/alkali vapor responsivity, RSC Advances, 4 (2014) 27281-27285.

[35] M.C. Chiappelli, R.C. Hayward, Photonic Multilayer Sensors from Photo-Crosslinkable Polymer Films, Adv. Mater. 24 (2012) 6100-6104.

[36] C. Lopez-Lopez, S. Colodrero, M.E. Calvo, H. Miguez, Angular response of photonic crystal based dye sensitized solar cells, Energy Environ. Sci. 6 (2013) 1260-1266.

[37] D.P. Puzzo, L.D. Bonifacio, J. Oreopoulos, C.M. Yip, I. Manners, G.A. Ozin, Color from colorless nanomaterials: Bragg reflectors made of nanoparticles, J. Mater. Chem. 19 (2009) 3500.

[38] C. Yao, J. Zhao, H. Ge, J. Ren, T. Yin, Y. Zhu, L. Ge, Fabrication of dual sensitive titania (TiO2)/graphene oxide (GO) one-dimensional photonic crystals (1DPCs), Colloids Surf. A: Physicochem. Eng. Asp. 452 (2014) 89-94.

[39] Z. Wang, J. Zhang, J. Xie, C. Li, Y. Li, S. Liang, Z. Tian, T. Wang, H. Zhang, H. Li, W. Xu, B. Yang, Bioinspired Water-Vapor-Responsive Organic/Inorganic Hybrid One-Dimensional Photonic Crystals with Tunable Full-Color Stop Band, Adv. Funct. Mater. 20 (2010) 3784-3790.

[40] L.D. Bonifacio, B.V. Lotsch, D.P. Puzzo, F. Scotognella, G.A. Ozin, Stacking the Nanochemistry Deck: Structural and Compositional Diversity in One-Dimensional Photonic Crystals, Adv. Mater. 21 (2009) 1641-1646.

[41] Y. Dou, J. Han, T. Wang, M. Wei, D.G. Evans, X. Duan, Fabrication of MMO-TiO2 one-dimensional photonic 
crystal and its application as a colorimetric sensor, J. Mater. Chem. 22 (2012) 14001-14007.

[42] M.E. Calvo, S. Colodrero, N. Hidalgo, G. Lozano, C. Lopez-Lopez, O. Sanchez-Sobrado, H. Miguez, Porous one dimensional photonic crystals: novel multifunctional materials for environmental and energy applications, Energy Environ. Sci. 4 (2011) 4800-4812.

[43] J. Ge, Y. Yin, Responsive Photonic Crystals, Angewandte Chemie International Edition, 50 (2011) 1492-1522.

[44] C. Yao, J. Ren, C. Liu, T. Yin, Y. Zhu, L. Ge, Hydrogel improved the response in the titania/graphene oxide one-dimensional photonic crystals, ACS Appl. Mater. Interfaces, 6 (2014) 16727-16733.

[45] S.Y. Choi, M. Mamak, G. von Freymann, N. Chopra, G.A. Ozin, Mesoporous Bragg Stack Color Tunable Sensors, Nano Lett. 6 (2006) 2456-2461.

[46] A.V. Dobrynin, M. Rubinstein, Theory of polyelectrolytes in solutions and at surfaces, Prog. Polym. Sci. 30 (2005) 1049-1118.

[47] J. Shin, S.G. Han, W. Lee, Dually tunable inverse opal hydrogel colorimetric sensor with fast and reversible color changes, Sens. Actuators, B. Chem. 168 (2012) 20-26. 


\section{Biographies}

Cihui Liu received his BS and MS degree from Nanjing University of Information Science and Technology in 2012. He is current a Ph.D candidate at State Key Laboratory of Bioelectronics, Southeast University. His research interests are focused on designing and fabricating bio-inspired intelligent interfacial materials and sensors using photonic band gap materials.

Chong Yao is currently a master student at the State Key Laboratory of Bioelectronics. He received his BS degree in Biomedical Engineering at Southeast University in 2012. His current research interests are focused on one-dimensional photonic crystals.

Yanxi Zhu is currently a Ph. D candidate at State Key Laboratory of Bioelectronics. He received his BS degree at LinYi University in 2010 and MS degree at GuiLin University in 2013. His current research interests are focused on self-healing materials.

Jiaoyu Ren is currently a master student at State Key Laboratory of Bioelectronics. She received his BS degree in Biomedical Engineering at Southeast University in 2013. Her current research interests are focused on one-dimensional photonic crystals.

Liqin Ge is a Professor of the Biological Science and Medical Engineering Department at Southeast University. She obtained her PhD degree at Institute of Chemistry Chinese Academy of Science in 2003. After post-doctoral periods in McGill University, she joined the faculty at Southeast University since 2005. Her research area covers the layer-by-layer self-assemble membranes, photonic band gap materials, and sensors using self-assembled polymers. 
Figure Captions

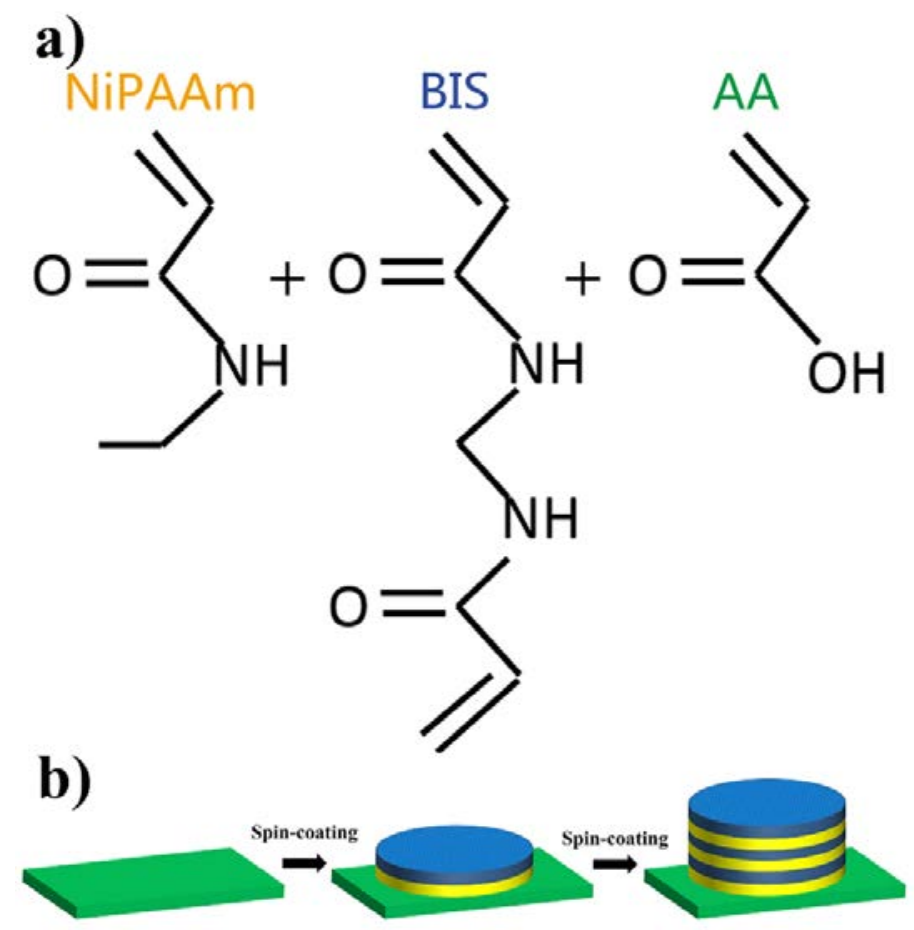

Figure 1. (a) Chemical structures of the NiPAAm, BIS and AA, (b) a schematic illustration of sensor fabrication, whereby polymer and $\mathrm{TiO}_{2}$ are sequentially spin-coated to create a multilayer $1 \mathrm{D}$ photonic stack. 
a)

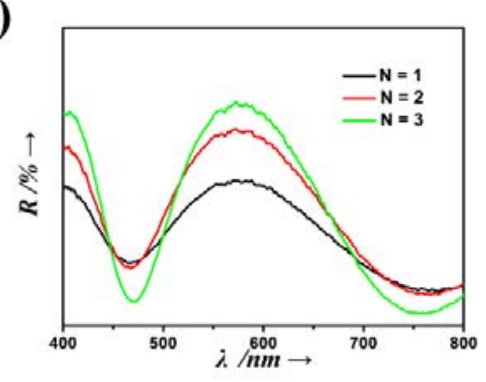

c)

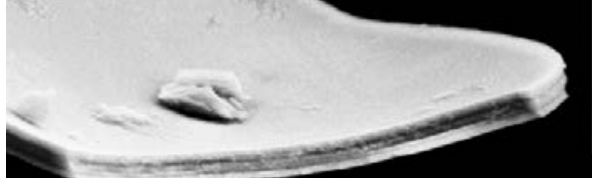

b)

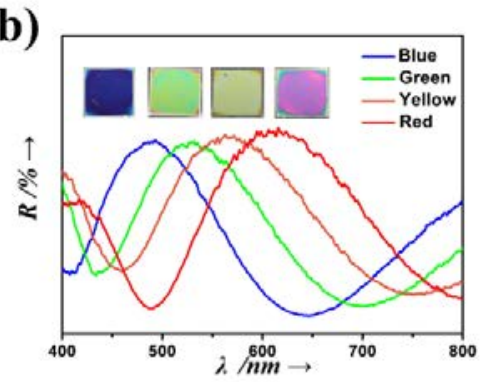

d)

$500 \mathrm{~nm}$

Figure 2. a) Reflection intensity of the 1DPCs with rising number of bilayers; b)

Structural color variation and reflection spectrum of the 1DPCs with different layer thickness; (c) shows the cross-section SEM image of a 1DPC of 6 layers, from which we can see multilayered structure of the 1DPC. (d) shows that both of the two materials are 3 layers, and the average thicknesses of polymer layer and $\mathrm{TiO}_{2}$ layer are $160 \mathrm{~nm}$ and $70 \mathrm{~nm}$ respectively. 
a)

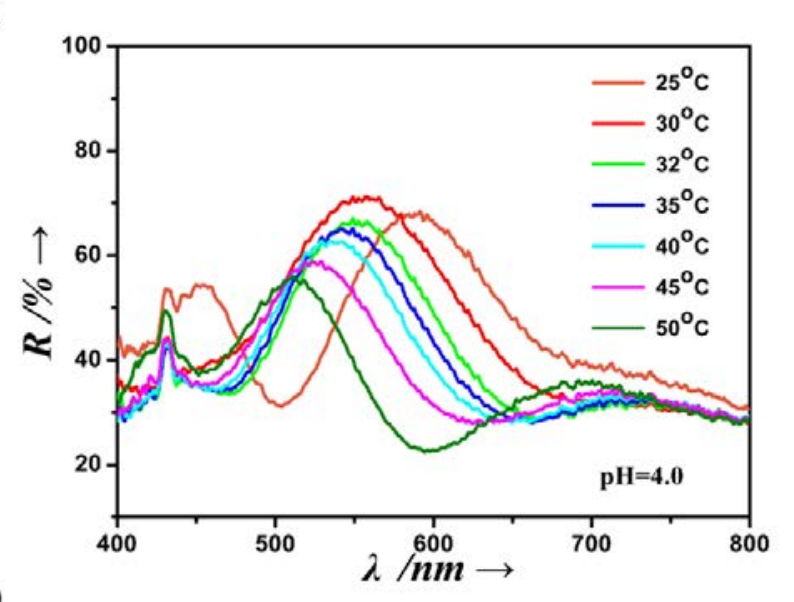

b)

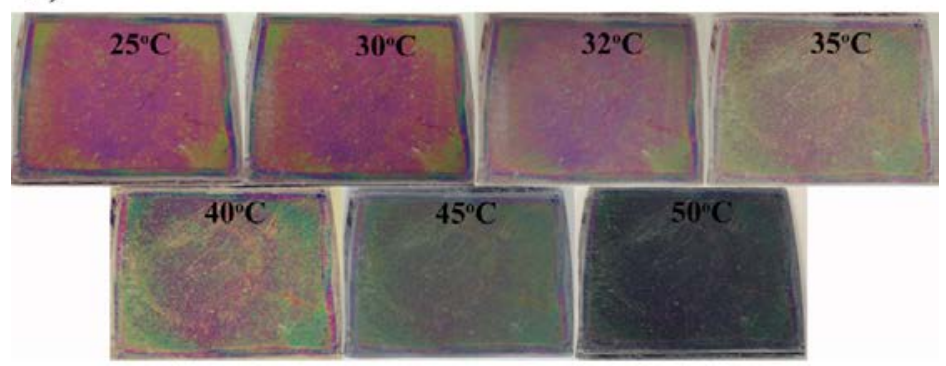

Figure 3. (a) The reflectance spectra of 1DPCs exposed to different temperatures varies from $25{ }^{\circ} \mathrm{C}$ to $50{ }^{\circ} \mathrm{C}$ under $\mathrm{pH}=4.0$, temperature $32{ }^{\circ} \mathrm{C}$ was specifically noted. (b) Photographs of the as-prepared 1DPCs corresponding to different temperatures. 


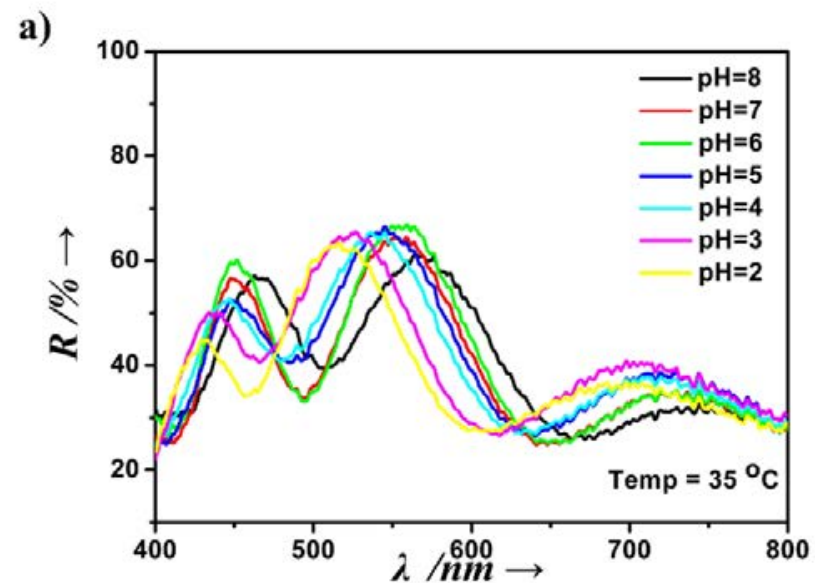

b)

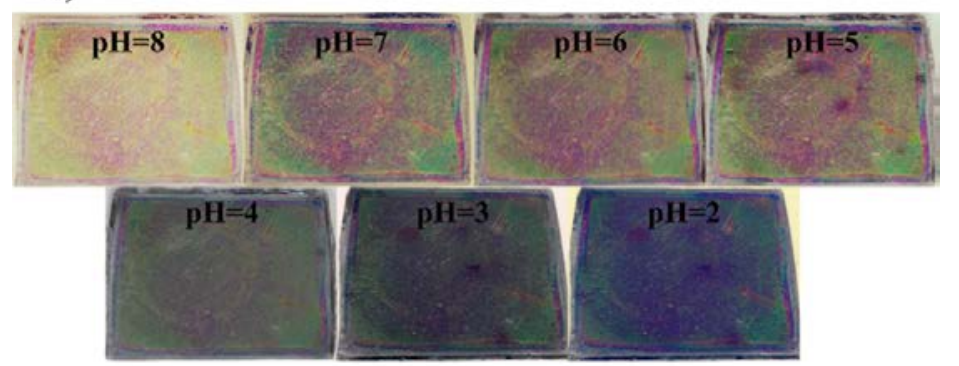

Figure 4. (a) The reflectance spectra of 1DPCs exposed to different $\mathrm{pH}$ varies from 8 to 2 under temperature $=35^{\circ} \mathrm{C}$. (b) Photographs of the as-prepared 1DPCs corresponding to different $\mathrm{pH}$. 

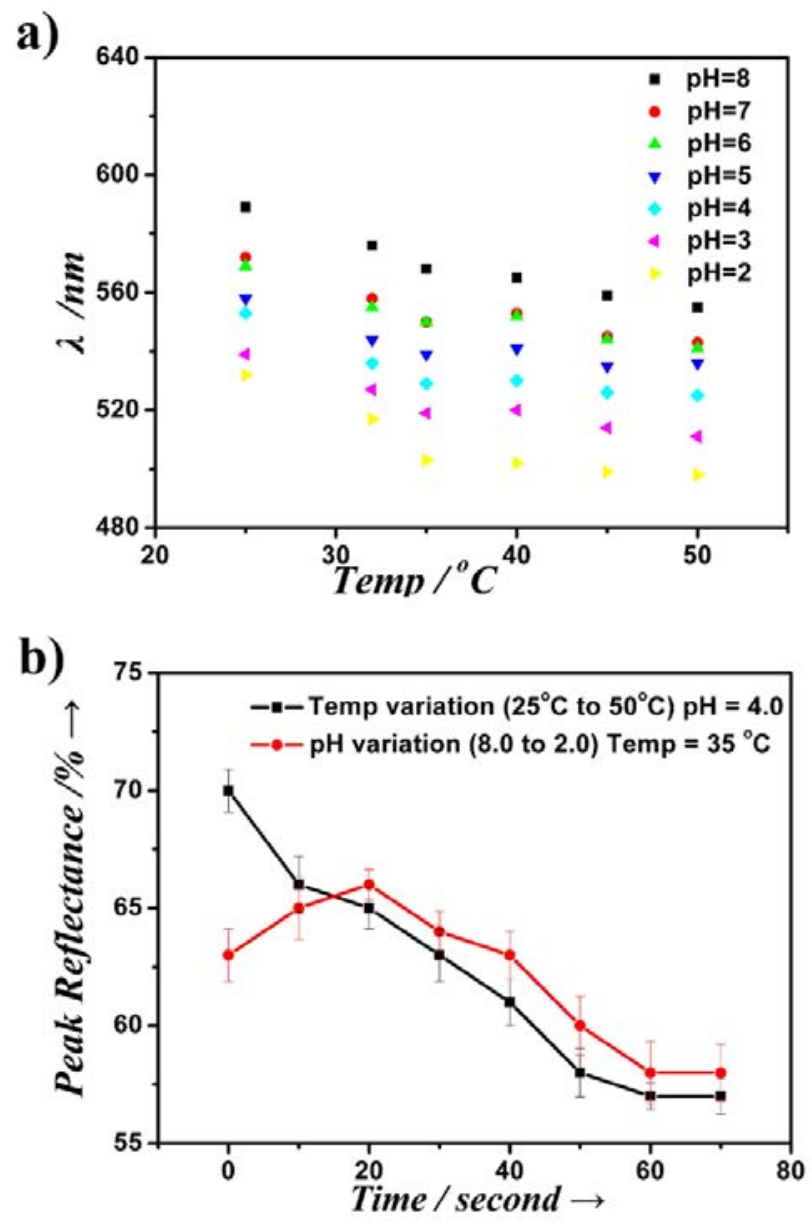

Figure 5. (a) Reflection maxima changes of a dual tunable 1DPC hydrogel sensor with respect to temperature variation measured at seven different pHs. (b) Plot of peak reflectance vs. time from the consecutive reflectance spectra obtained during $\mathrm{pH}$ change from 8 to 2 (red plots), and temperature from $25{ }^{\circ} \mathrm{C}$ to $50{ }^{\circ} \mathrm{C}$ (black plots). 
a)

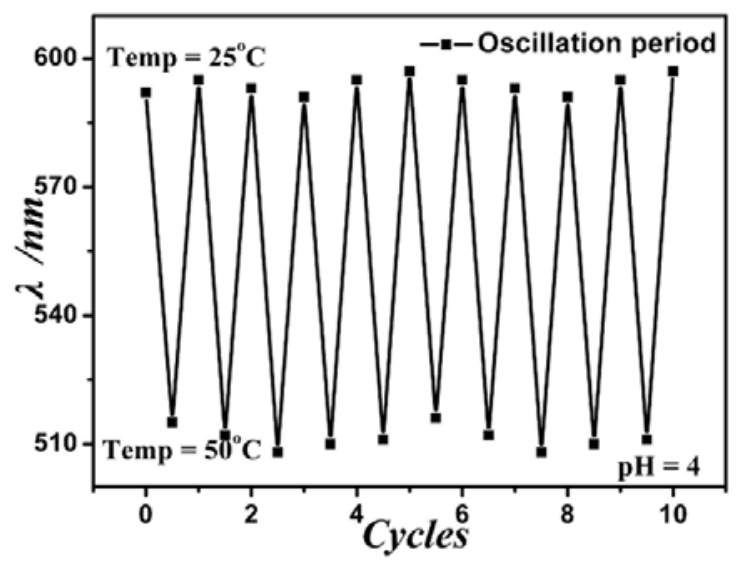

b)

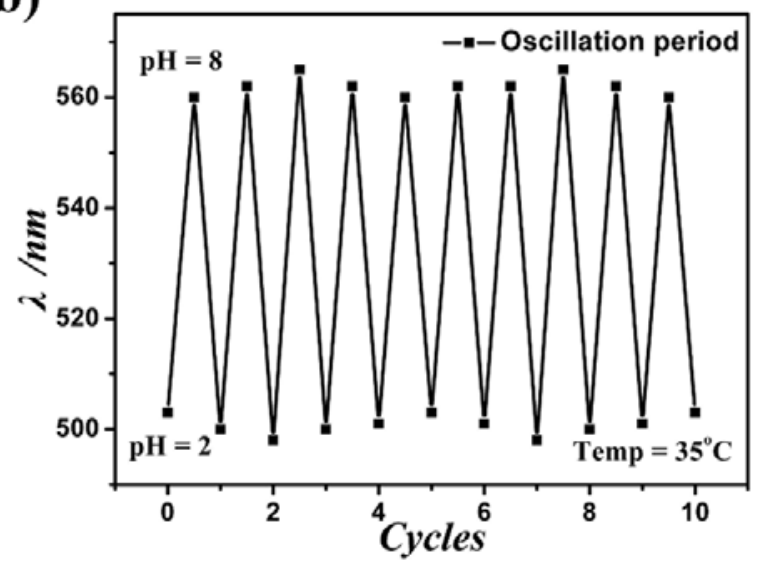

Figure 6. (a) 10 times reversible changes of the stopband of the 1DPC with temperature changes from $25{ }^{\circ} \mathrm{C}$ to $50{ }^{\circ} \mathrm{C}$ under $\mathrm{pH} 4.0$. (b) 10 times reversible changes of the stopband of the $1 \mathrm{DPC}$ with $\mathrm{pH}$ changes from 2 to 8 under temperature $35^{\circ} \mathrm{C}$. 

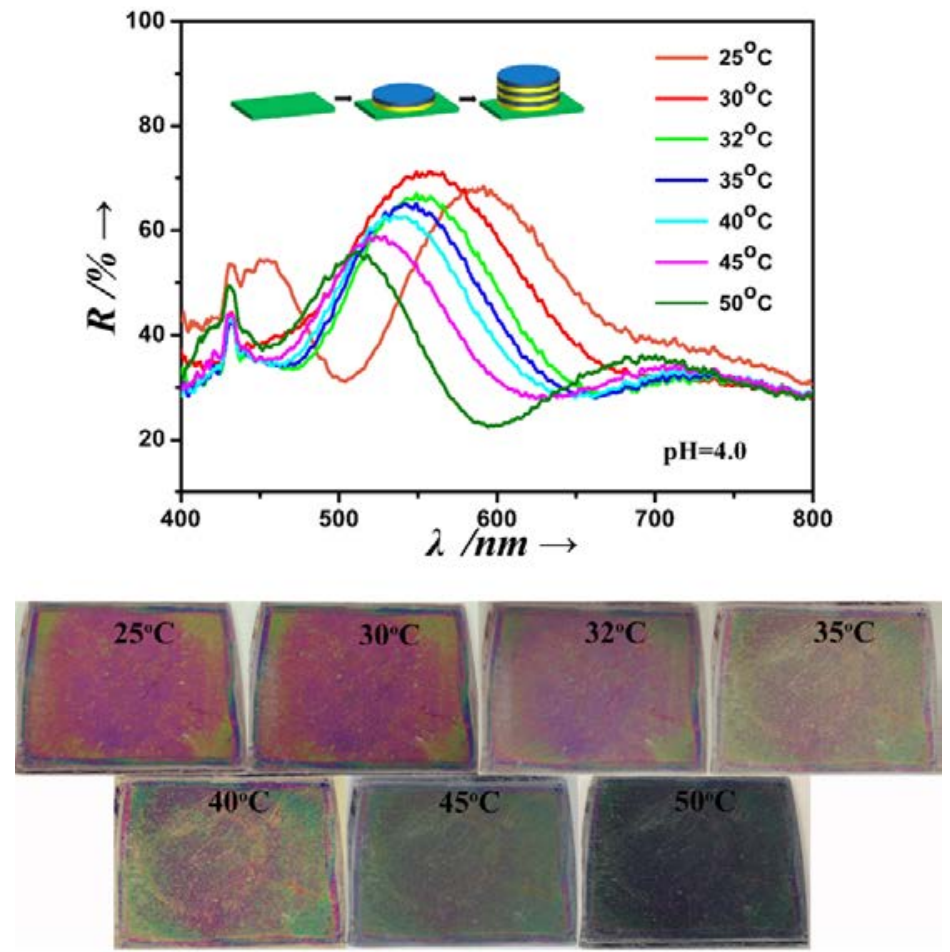

A smart and reproducible fabrication of $\mathrm{pH} /$ temperature dually responsive sensor by taking advantage of 1DPCs 